\title{
Detecting genomic regions associated with a disease using variability functions and Adjusted Rand Index
}

\author{
Dunarel Badescu, Alix Boc, Abdoulaye Baniré Diallo, Vladimir Makarenkov*
}

From Ninth Annual Research in Computational Molecular Biology (RECOMB) Satellite Workshop on Comparative Genomics

Galway, Ireland. 8-10 October 2011

\begin{abstract}
Background: The identification of functional regions contained in a given multiple sequence alignment constitutes one of the major challenges of comparative genomics. Several studies have focused on the identification of conserved regions and motifs. However, most of existing methods ignore the relationship between the functional genomic regions and the external evidence associated with the considered group of species (e.g., carcinogenicity of Human Papilloma Virus). In the past, we have proposed a method that takes into account the prior knowledge on an external evidence (e.g., carcinogenicity or invasivity of the considered organisms) and identifies genomic regions related to a specific disease.
\end{abstract}

Results and conclusion: We present a new algorithm for detecting genomic regions that may be associated with a disease. Two new variability functions and a bipartition optimization procedure are described. We validate and weigh our results using the Adjusted Rand Index (ARI), and thus assess to what extent the selected regions are related to carcinogenicity, invasivity, or any other species classification, given as input. The predictive power of different hit region detection functions was assessed on synthetic and real data. Our simulation results suggest that there is no a single function that provides the best results in all practical situations (e.g., monophyletic or polyphyletic evolution, and positive or negative selection), and that at least three different functions might be useful. The proposed hit region identification functions that do not benefit from the prior knowledge (i.e., carcinogenicity or invasivity of the involved organisms) can provide equivalent results than the existing functions that take advantage of such a prior knowledge. Using the new algorithm, we examined the Neisseria meningitidis FrpB gene product for invasivity and immunologic activity, and human papilloma virus (HPV) E6 oncoprotein for carcinogenicity, and confirmed some well-known molecular features, including surface exposed loops for N. meningitidis and PDZ domain for HPV.

\section{Background}

Many bacteria and viruses adapt to changing environmental conditions through several evolutionary mechanisms such as homologous recombination [1], nucleotide substitutions, insertions-deletions [2], horizontal gene transfer [3], etc. These mechanisms lead to the formation

\footnotetext{
* Correspondence: makarenkov.vladimir@uqam.ca Département d'Informatique, Université du Quebec a Montreal, C.P. 8888, Succursale Centre-Ville, Montreal (Quebec), H3C 3P8, Canada Full list of author information is available at the end of the article
}

of different polymorphic strands of the same group of organisms, in which the variation on the DNA composition is spread randomly throughout the genomes. The survival of these strands depends on their ability to overcome the environmental changes [4]. One of the goals of comparative genomics consists of finding the variation among aligned genomic sequences in order to identify functional regions. Several comparative genomic tools allow the identification of genomic regions in an alignment that have evolutionnary patterns different from the
C Biomed Central 
neutral evolution. For instance, PhastCons [5] predicts, from a given alignment and the related phylogenetic tree, the genomic regions under negative selection. PAML [6-8] allows the comparison of synonymous versus nonsynonymous mutations in an alignment in order to predict regions under selective pressure. RDP3 [9] and TOPAL [10] are software packages including several methods for detecting recombination. Most of these methods and software do not take into consideration external epidemiological evidence associated with many bacterial and virus strands. Such an evidence can allow the clustering of organisms based not only on the similarity of their genomic sequences, but also, on their association to different diseases. Hence, intra-specific and inter-specific variation among carcinogenic and noncarcinogenic human papilloma viruses can lead to the identification of regions related to carcinogenicity. In our previous works, we introduced a hit region identification function using prior knowledge information [11] and described the related validation framework based on Monte-Carlo simulations [12]. Then, we extended the latter study by presenting and testing four variants of the hit region identification function, still using the available prior knowledge [13]. In this paper, we present a new algorithm for the identification of specific genomic regions associated with an external disease. The introduced algorithm uses a bipartition optimization procedure to maximize a specific clustering function $Q$, based on inter- and intra-group variability, for each window position, over the given sequence alignment. It can be applied with or without prior knowledge information characterizing species in hand. Hit regions (i.e., putative regions related to a disease) can be validated using ARI [14] (a corrected-for-chance version of the Rand index [15]) and organismal bipartitions are constructed using the available epidemiological data. The new algorithm has been applied to two independent datasets: The human papilloma viruses and the Neisseria meningitidis data. The obtained results suggest that genomic regions with important biological features in both datasets can be associated with either carcinogenicity or invasivity.

\section{Dataset description}

\section{Neisseria meningitidis dataset}

Neisseria meningitidis is a Gram negative bacterium responsible for meningitis and septicemia. It has a relatively small genome size of $2.2 \mathrm{Mbp}$. In March 2011, the PubMLST database listed a total of 8,793 genetically distinct members of Neisseria organisms [16]. All these facts make N. meningitidis well suited for testing comparative genomics methods [17]. Proteins expressed under iron limitation (e.g. $\operatorname{FrpB}($ FetA)) are considered as potential vaccine components [18]. Bacteria grown under iron starvation express several proteins, the most abundant of them being FrpB, a 70kDa outer membrane protein (OMP). It is expressed in large amounts in all strains, and antibodies against this protein appear to be bactericidal. A putative FrpB topology was first proposed with a 26-stranded $\beta$-barrel [19], and later reassessed to a plug domain and a 22 -stranded $\beta$-barrel with 11 surface-exposed loops [20]. These loops are accessible to the host immune system, which produces natural antibodies against these regions. In general, bacteria express genetic sequence variability in order to evade this defense mechanism.

The data we considered, were classified on the invasivity basis using a list of identified hyperinvasive meningococci [21]. We then built a list of unique FetA sequence tags carried by the alleles of these organisms. Using local BLAST operations [22], we searched for the presence of these tags in the distinct sequences belonging to the selected multiple sequence alignment (MSA), first examined in [13]. We classified as belonging to the invasive category (subset $X$ ) any allele that contained at least one of the selected invasive tags. All the other alleles were put in the non-invasive category (subset $Y$ ). We annotated the MSA with the information regarding surfaceexposed loops, beta-sheets and periplasmic loops [20]. Translating indexes from the amino-acid sequences to DNA sequences were also computed. Each single value of the hit region identification function $Q$ (the $Q$-type functions will be used to identify genetic regions that may be related to a disease) corresponds to an interval of a certain length (i.e., 9 or 20 nucleotides in this study) and depends on the starting position of the sliding window used in our algorithm.

\section{Human papilloma virus dataset}

Human papilloma viruses (HPV) have a causal role in cervical cancer with almost half a million new cases occurring each year [23-25]. About a hundred of HPV types have been identified, and the whole genomes of more than eighty of them have been sequenced (see the latest Universal Virus Database report by International Committee on Taxonomy of Viruses (ICTV)). A typical HPV genome is a double-stranded, circular DNA genome of size close to $8 \mathrm{Kbp}$, with a small set of genes (L1, L2, E1, E2, E4, E5, E6 and E7). In this study, we focused on the gene E6, which is predominantly linked to cancer due to the binding of its product to the p53 tumor suppressor protein. It contains a PDZ domainbinding motif $(-\mathrm{X}-\mathrm{T}-\mathrm{X}-\mathrm{V})$ at its carboxy terminus, which is essential for targeting the PDZ proteins for proteasomal degradation. Such proteins include hDlg, hScrib, MAGI-1, MAGI-2, MAGI-3 and MUPP1 [26]. The interaction between E6 and hDlg, or the other PDZ domain-containing proteins, may be an underlying mechanism in the development of HPV-associated 
cancers [27]. The gene E6 was also shown to contain two stable folded domains, E6N and E6C [28,29]. Models of these domains have been built in the absence of complete crystallographic data [30].

To define carcinogenic types, we used the epidemiological data from a large international survey on HPV in cervical cancer and from a multicenter case-control study conducted on 3,607 women with histologically confirmed cervical cancer [31,32]. More than $89 \%$ of them had squamous cell carcinoma (i.e., Squam cancer) and about $5 \%$ had adenosquamous carcinoma (i.e., Adeno cancer). More than a half of the infection cases were due to the types 16 and 18 of HPV, which are later referred to as High-Risk HPV [33]. In this study, we examined the content of the gene E6 for 83 different HPV types.

We fixed the window size to 20 nucleotides for HPV datasets in order to be consistent with our previous works $[11,12]$, where we conducted simulations with windows of different sizes and used the size of $20 \mathrm{bp}$ to present the results. In the same way, we considered the window size of 9 nucleotides for the $N$. meningitidis dataset to be consistent with another our study [13].

\section{Methods}

\section{Description of the algorithm}

The new algorithm takes as input a MSA established for a set of organisms. Assume that this set of organisms is partitioned into two different subsets according to a boolean criterion (e.g., invasivity vs. non-invasivity or carcinogenicity vs. non-carcinogenicity). The corresponding subsets are denoted $X$ (invasive/carcinogenic) and $Y$ (non-invasive/non-carcinogenic), respectively. The region of interest is scanned using a non-overlapping sliding window, as shown in Figure 1, of a fixed width (20 sites for HPV and 9 sites for $N$. meningitidis). For each window position, we carry out a bipartition optimization algorithm in order to search for maximum values of the hit region identification function. A specific version of the Q-type function (see below) can be taken as the

\footnotetext{
18), all the other sequences belong to the set $Y$ (non-carcinogenic HPV). The HPV type is indicated in the left column.
}

algorithm parameter. We denote by $Q^{\prime}$ a specific version of the Q-type function computed under condition that the subsets bipartition is unknown (i.e., prior knowledge). The complete algorithmic scheme is presented in Algorithm 1 in Additional file 1 .

\section{Clustering using the Q-type functions}

To perform the clustering of our data into two groups $A$ and $B$, we first calculate the intragroup variability of the sequences from the group $A$, denoted by $V(A)$, the group $B$, denoted by $V(B)$, and, finally, the intergroup variability $D(A, B)$, as described in Equations 1, 2 and 3. These measures are defined as the means of the squared Hamming distances, dist, among the sequence fragments (bounded by the sliding window position) of the taxa from the group $A$ only, from the group $B$ only, and between the sequence fragments from the distinct groups $A$ and $B$ :

$$
\begin{aligned}
& V(A)=\frac{1}{N(A)(N(A)-1) / 2} \sum_{\left\{a_{1}, a_{2} \in A \mid a_{1} \neq a_{2}\right\}} \operatorname{dist}_{h}^{2}\left(a_{1}, a_{2}\right), \\
& V(B)=\frac{1}{N(B)(N(B)-1) / 2} \sum_{\left\{b_{1}, b_{2} \in B \mid b_{1} \neq b_{2}\right\}} \operatorname{dist}_{h}^{2}\left(b_{1}, b_{2}\right), \\
& D(A, B)=\frac{1}{N(A) N(B)} \sum_{\{a \in A, b \in B\}} \operatorname{dist}_{h}^{2}(a, b) .
\end{aligned}
$$

In [11-13] four different hit region identification functions, $Q_{1}, Q_{2}, Q_{3}$ and $Q_{4}$, which could be summarized by the following equation, were defined:

$$
Q=D(A, B)-k \times V(A)-l \times V(B),
$$

where the $[k, l]$ combinations are as follows: $\left\{Q_{1} \mapsto(1,0), Q_{2} \mapsto(0,1), Q_{3} \mapsto\left(\frac{1}{2}, \frac{1}{2}\right), Q_{4} \mapsto(0,0)\right.$.

The function $Q_{4}$ (Equation 5), along with new versions of the hit region identification function, denoted by $Q_{5}$ (Equation 6) and $Q_{6}$ (Equation 7), will be tested and discussed in this study:

$$
\begin{aligned}
& Q_{4}=D(A, B), \\
& Q_{5}=|V(A)-V(B)|, \\
& Q_{6}=|V(A) / V(B)| .
\end{aligned}
$$

\section{Measuring the agreement between the reference and the optimal calculated bipartitions using the Adjusted Rand Index (ARI)}

The Adjusted Rand Index [14] has become a criterion of choice for measuring agreement between two partitions 
in clustering analysis [34]. Having a calculated bipartition $U^{\prime \prime}=A \mid B$ and a reference bipartition $U^{\prime}=X \mid Y$, for all $\left(\begin{array}{c}n \\ 2\end{array}\right)$ pairs of elements, one can compute how many of them fall into the same group and how many in different groups. One can then calculate ARI [35] according to Equation 8. ARI is the corrected-for-chance version of the Rand index [15]. It ranges between -1 and 1 , and expresses the level of concordance between two bipartitions [14]. The values of ARI close to 1 indicate an almost perfect concordance between the two compared bipartitions, whereas the values close to -1 indicate a complete discordance between them:

$$
A R I=\frac{\left(\begin{array}{l}
n \\
2
\end{array}\right)(a+d)-[(a+b)(a+c)+(c+d)(b+d)]}{\left(\begin{array}{l}
n \\
2
\end{array}\right)^{2}-[(a+b)(a+c)+(c+d)(b+d)]},
$$

where $\left(\begin{array}{l}n \\ 2\end{array}\right)=a+b+c+d, a$ is the number of pairs that are in the same group in the bipartitions $U^{\prime \prime}$ and $U$ ${ }^{\prime}, b$ is the number of pairs that are in the same group in the bipartition $U^{\prime \prime}$ and in different groups in the bipartition $U^{\prime}, c$ is the number of pairs that are in different groups in the bipartition $U^{\prime \prime}$ and in the same group in $U$ ', and $d$ is the number of pairs that are in different groups in the bipartitions $U^{\prime \prime}$ and $U^{\prime}$.

\section{Validation of the obtained hit regions using the Adjusted Rand Index}

We define a new function $Q^{\prime \prime}$ reflecting the quality of the reference bipartition, as follows:

$$
Q^{\prime \prime}=A R I \times Q^{\prime} .
$$

The difference between $Q^{\prime}$ and $Q^{\prime \prime}$ indicates the level of concordance of the reference bipartition $U^{\prime}$ with the selected function $Q$. Throughout the paper, $Q$ will denote the hit identification function using prior knowledge information, $Q^{\prime}$ - not using any prior knowledge information and $Q^{\prime \prime}$ - using prior knowledge information and based on ARI.

\section{Bipartition optimization}

For each window position, we generated a fixed number of random initial bipartitions. For each such a bipartition, we moved elements from one subset to the other and back again in cycles, each time accepting the move that maximized the objective function $Q$, until no further improvement was possible. Once a local maxima was reached, we compared it to the best current value obtained for all starting random bipartitions tested so far. ARI was used to compare the level of concordance of the obtained bipartition (i.e., the one that was maximizing the given function $Q$ ) with the reference bipartition (carcinogenic vs. non-carcinogenic taxa for HPV and invasive vs. non-invasive taxa for $N$. meningitidis) given as a parameter to the algorithm.

\section{Time complexity}

The time complexity of the new algorithm carried out with an overlapping sliding window of a fixed width, and advancing one alignment site by step, is $O\left(l \times n^{2} \times\right.$ $w \times r$ ), where $l$ is the length of the MSA, $n$ the number of considered species, $r$ the number of random initial partition generations and $w$ the window width. In order to ensure this complexity, we have to limit the optimization cycle to a constant number of iterations.

\section{Simulation study}

In order to validate the hit region identification functions $Q_{5}^{\prime}, Q_{5}^{\prime}$ and $Q_{6}^{\prime}$, we conducted a Monte-Carlo simulation study involving two major evolutionary mechanisms: Positive selection (PS) and Lineage specific selection (LSS). Two cases of group selection were also tested: The cases of the monophyletic and polyphyletic clustering. An approach involving the computation of p-values was implemented to asses the predictive ability of each of the three functions for each combination of evolutionary parameters. The following procedure was carried out. A phylogenetic tree $T$ with 16 leaves was first generated using the algorithm described by Kuhner and Felsenstein [36]. The edge lengths of $T$ were generated using an exponential distribution. Following the approach of Guindon and Gascuel [37], we added some noise to the tree edges in order to provide a deviation from the molecular clock hypothesis. The random trees yielded by this procedure had depth of $O(\log (16))$. The tree was then rooted by midpoint. For the monophyletic test, the left and right sub-trees, denoted by $T_{1}$ and $T_{2}$, were determined, depending on the position of the root. For the polyphyletic test, two sets of leaves were randomly chosen and the corresponding sub-trees, denoted by $T_{3}$ and $T_{4}$, were extracted.

In the PS simulations, we used the original lengths of the edges of the subtrees $T_{1}$ and $T_{2}$ (i.e., monophyletic case), and $T_{3}$ and $T_{4}$ (i.e., polyphyletic case), while all edge lengths of $T$ were gradually multiplied by the scaling factor $a$, varying from 0.05 to 1 (with the step of 0.05).

In the LSS simulations, all edge lengths of $T$ were multiplied by 0.5 (thus simulating neutral evolution), while all edge lengths of $T_{1}$ and $T_{3}$ were multiplied by the scaling factor $a_{1}=0.5+0.025 x$, and all edge lengths of $T_{2}$ and $T_{4}$ by $a_{2}=0.5-0.025 x$, where $x$ was varying from 1 to 19 .

Second, we executed the SeqGen program [38] to generate random MSAs of nucleotide sequences along the edges of the phylogenetic trees constructed at the first step. The SeqGen program was used with the Jukes-Cantor model of sequence evolution [39]. DNA sequences with $440 \mathrm{bp}$ were generated for each tree $T$. In addition, MSAs of the length 20 bp were generated for each of the 
trees $T_{1}, T_{2}, T_{3}$ and $T_{4}$. Two different variants of MSA were produced to simulate monophyletic and polyphyletic evolution. In the sequence alignment generated for the original tree $T$, we inserted those generated for the trees $T_{1}$ and $T_{2}$ in the monophyletic case, and those generated for the trees $T_{3}$ and $T_{4}$ in the polyphyletic case. The location of the inserted sequence blocks was known.

Thus, depending on the scaling factor parameters, for the PS case we simulated a variable homogeneous region inside a conserved context, and for the LSS case a more divergent region inside a neutral context. Third, we scanned the resulting sequence alignment using a sliding window of size $20 \mathrm{bp}$ with the step of 1 . We calculated the value of the hit region identification functions $Q_{4}^{\prime}, Q_{5}^{\prime}$ and $Q_{6}^{\prime}$ for each fixed position of the window and assessed the proportion of their values that were higher than the reference value corresponding to the inserted region.

These steps were repeated over 100 different replicates and the distributions of the best (in each case) function over each combination of testing parameters were represented using quartiles.

\section{Results and discussion}

We proposed a new algorithm for finding genomic regions that may be related to a disease along with two new hit region identification functions $Q_{5}$ and $Q_{6}$. Both new functions along with the best existing function $Q_{4}$ were tested in simulations. The functions yielding the best results for each case were illustrated in Figure 2: Monophyletic evolution (case a: PS, case b: LSS) and Figure 3: Polyphyletic evolution (case a: PS, case b: LSS). The remaining results for the $Q_{4}^{\prime}, Q_{5}^{\prime}$ and $Q_{6}^{\prime}$ functions are presented in Additional file 1. Figures 2 and 3 clearly show that the hit zone identification in the monophyletic case is much easier than in polyphyletic case. We can suggest that in order to be recognized, the hit region has to have a different evolutionary speed than the context in which it resides. The polyphyletic lineage specific case represents the hardest evolutionary situation. Also, one can notice that different $Q$-type functions, $Q_{4}^{\prime}, Q_{5}^{\prime}$ or $Q_{6}^{\prime}$, should be used in different practical situations.

The procedure for the identification of hit regions was carried out to detect the variability zones in the FrpB gene of $N$. meningitidis as well as the regions potentially responsible for cancer in the gene E6 of HPV. In both cases, we also carried out the ARI validation.

\section{Neisseria meningitidis analysis}

We scanned the MSA of the FrpB gene using the new algorithm with a sliding window of size 9 nucleotides. We compared the obtained results to the putative

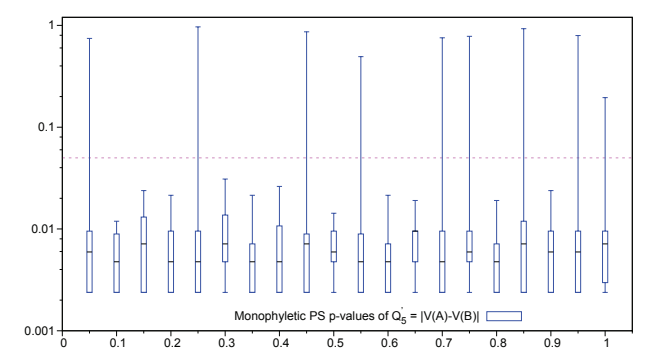

(a)

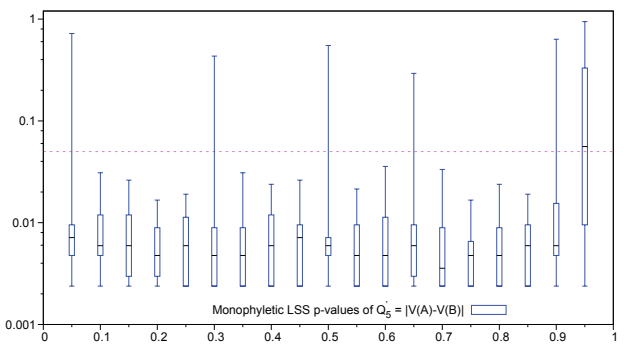

(b)

Figure 2 p-values obtained for monophyletic evolution hit region detection (a) Positive selection - Variable hit region inside conserved context. Quartile distribution of $p$-values obtained for the function $Q_{5}^{\prime}$. Abscissa represents scaling factor of the conserved context in which the variable hit region resides. Values close to 0 represent conservation (maximum discrimination), while values close to 1 represent variability (identical to context). Variable hit region is always maintained at a scaling factor of 1 . Ordinate represents $p$ values in log-scale. Horizontal dashed line represents the significance threshold of 0.05 . (b) Lineage specific selection Heterogeneous hit region inside neutral context. Quartile distribution of p-values obtained for the function $Q_{5}^{\prime}$. Abscissa represents the difference in scaling factors among the two lineages present in the hit region. Values close to 0 represent homogeneous evolutionnary speed (similar to the neutral context in which it resides), while values close to 1 represent divergence among these lineages. Context is always maintained at a scaling factor of 0.5, simulating neutral evolution. Horizontal dashed line represents the significance threshold of 0.05 . In the case of lineage specific selection, the value of the $Q^{\prime}$-type functions corresponding to 1 on the abscissa scale cannot be computed because it involves a subtree with 0 edge lengths.

topology model of the FrpB protein described in [20] (see Figure 4a). The results are presented in Figure 4b and $\mathrm{c}$.

Remarkably, all surface exposed loops confirmed by enzyme-linked immunosorbent assay (i.e., L2, L3, L4, L5 and L10) [20] were properly detected using the functions $Q_{4}^{\prime}$ and $Q_{5}^{\prime}$. It is worth noting that our algorithm was able to find the loop L4, which is hidden between the loops L5 and L3. The model loops L1, L8 and L9 were found at their predicted positions. The loops L2 and L11 were found at different positions, while the loops L6 and L7 were missed regardless of the availability of the prior 


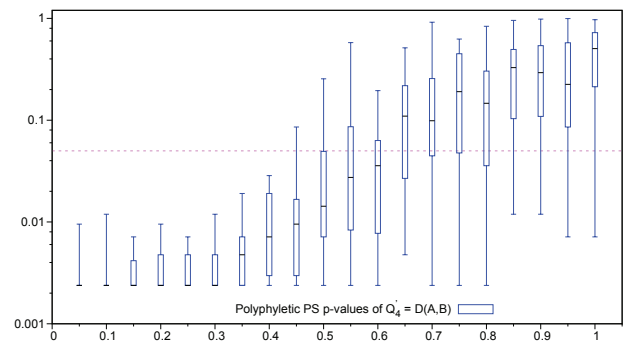

(a)

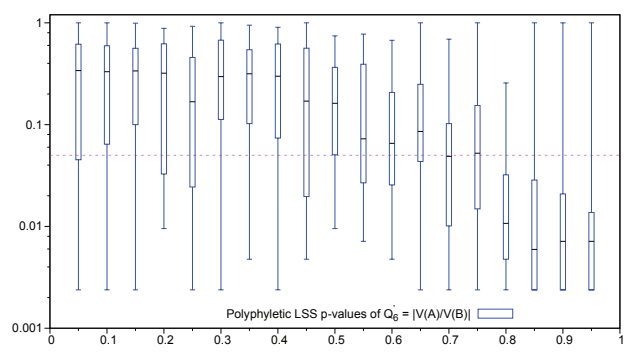

(b)

Figure 3 p-values obtained for polyphyletic evolution hit region detection (a) Positive selection - Variable hit region inside conserved context. Quartile distribution of $p$-values obtained for the function $Q_{4}^{\prime}$. Variable hit region is always maintained at a scaling factor of 1. Abscissa represents scaling factor of the conserved context in which the variable hit region resides. Values close to 0 represent conservation (maximum discrimination), while values close to 1 represent variability (identical to context). Ordinate represents p-values in log-scale. Horizontal dashed line represents the significance threshold of 0.05. (b) Lineage specific selection Heterogeneous hit region inside neutral context. Quartile distribution of p-values obtained for the function $Q_{6}^{\prime}$. Context is always maintained at a scaling factor of 0.5 , simulating neutral evolution. Abscissa represents difference in scaling factors among the two lineages present in the hit region. Values close to 0 represent homogeneous evolutionnary speed (similar to the neutral context in which it resides), while values close to 1 represent divergence among these lineages, and from the neutral context. Horizontal dashed line represents significance threshold of 0.05 .

knowledge information (see Figure $4 \mathrm{~b}$ and c). As protein models gradually improve and more crystallographic data become available, it will be interesting to reassess these results in the future. Both presented $Q^{\prime}$-type functions (Equations 5-6) overlap along the alignment, with the exception of the largest loop (L5) and the second largest loop (L3), where the amino acid variability is largely confined. The function $Q_{4}^{\prime}$ correlates best with surface exposed loops structure. This suggests that the divergences in shape between the functions $Q_{4}^{\prime}$ and $Q_{5}^{\prime}$ might be used to detect immunologic activity. It is known that bactericidal antibodies are directed against variable regions situated in the largest loops of proteins [40]. Note that the organisms compared here were strains of the same bacterium; their genetic variant being alleles and evolutionary distances between them being very small. On such a small timescale, underlining evolutionary processes are usually not very diverse. It would be also interesting to verify whether similar conclusions could be made for other outer membrane proteins.

\section{Human Papilloma Virus analysis}

We performed a scan of the MSA of the gene E6 for 83 HPV organisms (using non-overlapping windows of size 20 nucleotides). Each time the species bipartition was known, High-Risk HPV against all other HPV types in Figure 5a, Squam-Risk HPV against all other HPV types in Figure 6a, and Adeno-Risk HPV against all other HPV types in Figure 6b, it was incorporated in the computational procedure as shown in Algorithm 1. The comparative results for the High Risk HPV subset provided by the new algorithm without prior knowledge of carcinogenic taxa and those yielded by the former one [11], are presented in Figure 5 using annotations for HPV-16. Figure 5a illustrates the results obtained using the functions $Q_{4}$ and $Q_{5}$ using a prior knowledge on the species carcinogenicity.

According to the new algorithm, see Figure 5b, the PDZ domain is ranked first in the annotated part of the alignment. A detailed view of the terminal aligned region, within the index interval 680-740, shows a small left shift in the peak positions of the function $Q_{4}^{\prime}(5 b)$, but inside the same C-terminal tail domain. On the left side, flanking the PDZ domain, one can find the E6C domain which is related to the DNA binding [30]. One can notice that the function peaks (see Figure $5 \mathrm{a}$ and $5 \mathrm{~b})$ of $Q_{4}^{\prime}$ are almost in the same positions than those found using $Q_{4}$, exception being a region at the beginning of the alignment (i.e., at the beginning of the E6N domain). As for $N$. meningitidis loops, it would be interesting to study in greater details the regions recognized by both tested functions, $Q_{4}^{\prime}$ and $Q_{5}^{\prime}$.

We can conclude, by comparing Figures $5 \mathrm{a}$ and $5 \mathrm{~b}$, that the new functions, $Q_{4}^{\prime}$ and $Q_{5}^{\prime}$, provide almost identical hit region recovery than the existing functions $Q_{4}$ and $Q_{5}$, which take advantage of a prior knowledge on the species carcinogenicity.

The $Q^{\prime \prime}$ function validation was also carried out for HPV data. The results are presented in Figure 6. Here, the PDZ domain ranks first for both tested datasets, related to the Squam and Adeno cancers (Figures 6a and $6 \mathrm{~b}$ ). The peaks were found at almost the same positions as in Figure 5, with the exception that only some of the peaks shown in Figure 5 are present here. The function $Q_{4}^{\prime \prime}$ seems to be less variable than the function $Q_{5}^{\prime \prime}$. For the Squam dataset, there is one peak in the E6C domain, absent in the Adeno dataset, with a high monophyletic signal and unknown annotation. 


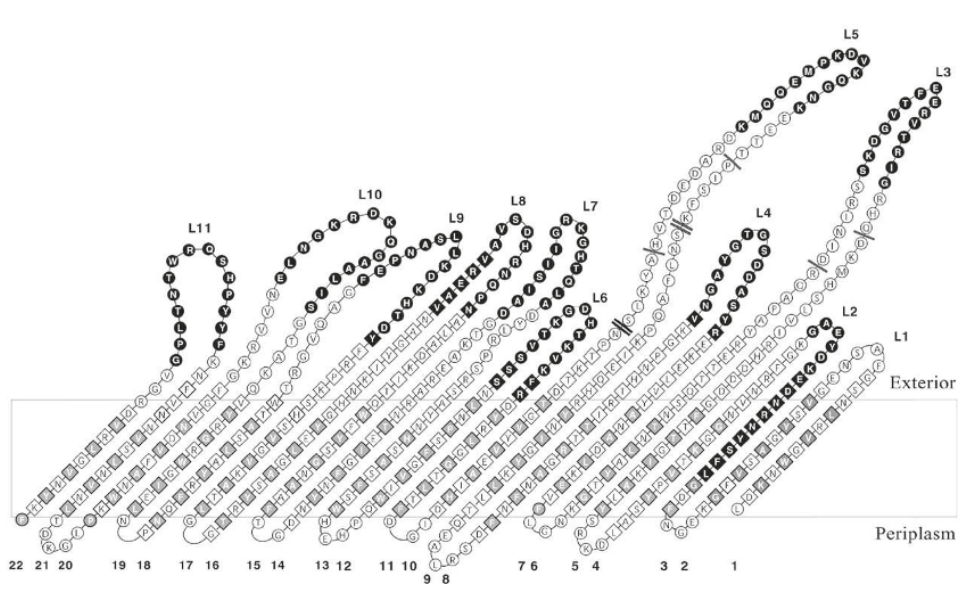

(a)

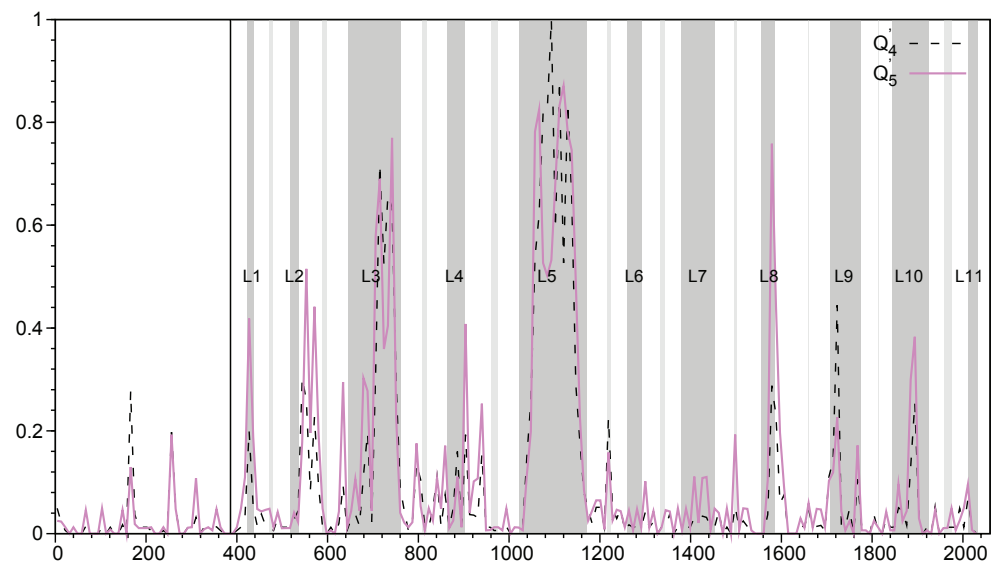

(b)

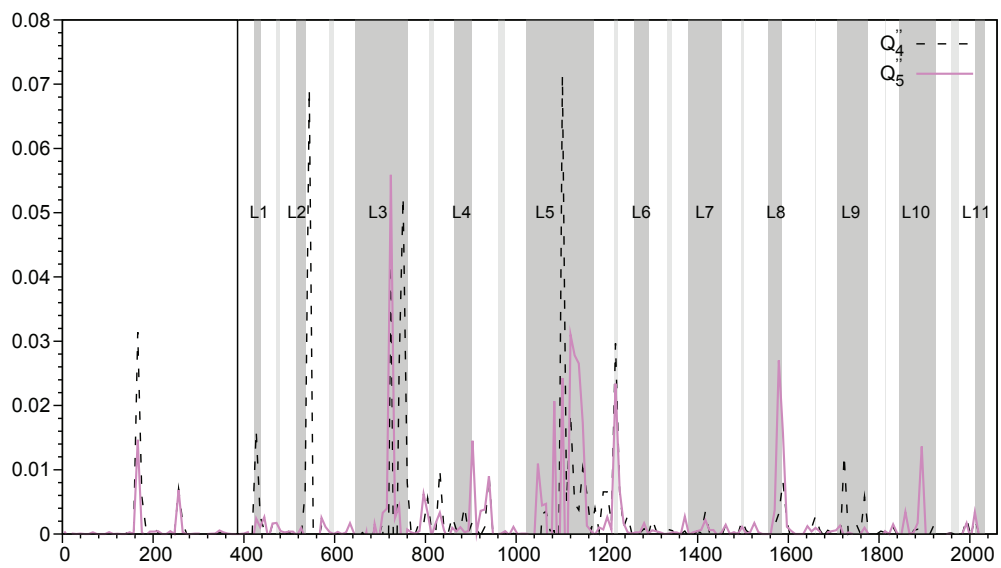

(c)

Figure $4 \mathbf{N}$. meningitidis FrpB protein variability zone detection (a) Topology model of the FrpB protein of N. meningitidis strain H44/76. Topology of the $\beta$-barrel. Surface-exposed loops $(\mathrm{L})$ and $\beta$-strands are numbered. Residues are framed according to their predicted secondary structure: Amino acid residues in $\beta$-strands are depicted by diamonds. Amino acid residues present in exposed loops and periplasmic turns are depicted by circles (reproduced from Kortekaas et al., 2007) [20]. (b)-(c) Variability zone detection by the hit region identification $Q^{\prime}-$ type functions, achieved without prior knowledge of invasive taxa (case b), and Q"-type functions, using this prior knowledge along with the ARI coefficient (case c). Functions $Q_{4}^{\prime}$ and $Q_{4}^{\prime \prime}$ are depicted by a dashed line and functions $Q_{5}^{\prime}$ and $Q_{5}^{\prime \prime}$ are depicted by a continuous line. A nonoverlapping sliding window of size 9 nucleotides was used during the scan of the gene FrpB MSA. The abscissa axis represents the window position along the nucleotide MSA. 11 gray zones correspond to extracellular loops. Annotations start at the solid vertical line (near the 400 abscissa mark). 


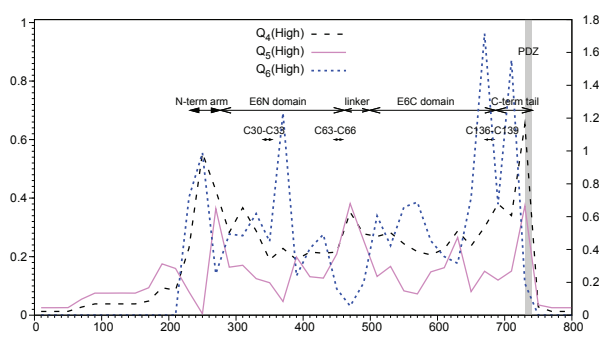

(a)

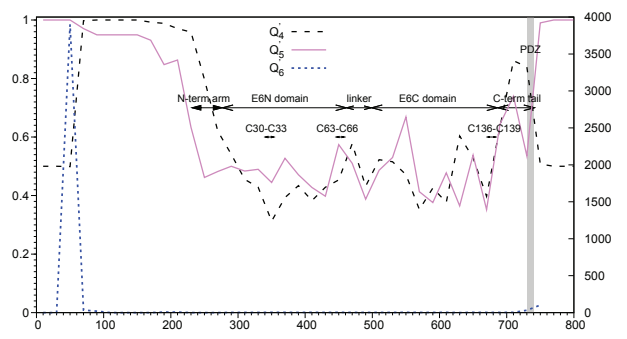

(b)

Figure 5 Hit region identification functions for High-Risk HPV (a) Functions obtained using prior knowledge on the taxa carcinogenicity. The hit region identification functions $Q_{4}$, depicted by a dashed line, $Q_{5}$, depicted by a continuous line, and $Q_{6}$, depicted by a dotted line, for the High-Risk HPV (HPV-16 and 18) $[11,12]$, during the scan of the gene E6. (b) Functions computed without prior knowledge on the taxa carcinogenicity. The hit region identification functions $Q_{4}^{\prime}$, depicted by a dashed line, $Q_{5}^{\prime}$, depicted by a continuous line, and $Q_{6}^{\prime}$, depicted by a dotted line, during the scan of the gene E6. The abscissa axis represents the window position along the nucleotide multiple sequence alignment. The PDZ-doirmm is highlighted in gray. Annotations for the $\mathrm{N}$ and Cterminal arms, E6N and E6C domains are represented for HPV16 coordinates, from (Nominé et al., 2006) [30]. Zn ${ }^{2+}$-ligating Cys residues annotations reproduced from Lipari et al., 2001 [28].

On the other hand, the peak located at the index 660, and corresponding to the window positions 660-680, includes two putative $\mathrm{Zn}^{2+}$-ligating Cys residues whose absence in mutants results in a dramatic loss in the p53 degradation activity [28].

By analyzing Figures 4, 5 and 6, one can notice that in some situations prior knowledge information brings an important advantage to the method (see the case of Figure 4c when the use of the prior knowledge along with the ARI coefficient allows for getting rid of some false positive hits; for instance, the false positive picks found using $Q^{\prime}$-t type functions around the indices 1225 and 1500 presented in Figure $4 \mathrm{~b}$ were not found by the $Q^{\prime \prime}$-type functions presented in Figure $4 \mathrm{c}$ as well as the case of an almost perfect PDZ domain recovery provided by the $Q^{\prime \prime}$-type functions as shown in Figures 6a and $6 \mathrm{~b}$ ), but in the other cases, the new algorithm is capable of correct recovering hit regions without any

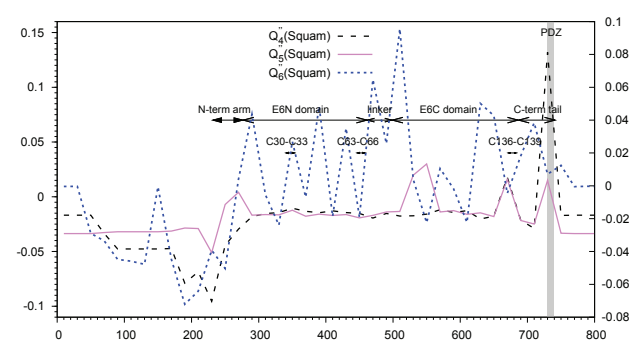

(a)

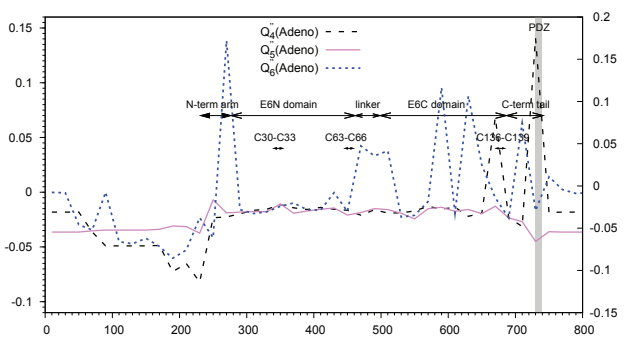

(b)

Figure 6 Q"-type functions, depending on ARI (a) Squam HPV dataset. (b) Adeno HPV dataset. Variation of the function $Q^{\prime \prime}{ }_{4}$, depicted by a dashed line, $Q^{\prime \prime}{ }_{5}$, depicted by a continuous line, and $Q_{6}^{\prime \prime}$, depicted by a dotted line, obtained with the non-overlapping sliding window of width 20 nucleotides during the scan of the gene E6. The abscissa axis represents the window position along the nucleotide MSA. The PDZ-domain is highlighted in gray. Annotations for the $\mathrm{N}$ and C-terminal arms, E6N and E6C domains are represented for HPV16 coordinates, from (Nominé et al., 2006) [30]. $\mathrm{Zn}^{2+}$-ligating Cys residues annotations reproduced from Lipari et al., 2001 [28].

prior knowledge (e.g., see the cases of the loops L1, L3, L5, L8, L9 and L10 for the N. meningitidis dataset).

\section{Conclusion}

We described a new algorithm for finding genomic regions that may be associated with a disease. It is capable of detecting hit regions without prior knowledge on the carcinogenicity or invasivity of related organisms. This is an important improvement over previous works in the field [11-13]. We also showed as the Adjusted Rand Index $[14,34,35]$ can be incorporated in the hit detection procedure. The discussed algorithm can be directly used to study organisms that have an ambivalent behavior and are, thus, more difficult to classify. For instance, some strains of Neisseria Meningitidis show a hyperinvasive behavior during epidemics, but are noninvasive, otherwise. The behavior of some other organisms, like human papilloma viruses (HPV), is more consistent. Such organisms can be clearly classified with respect to their level of carcinogenicity. Species bipartitions, established according to a carcinogenicity or invasivity criterion, suggested in the literature are important 
for the identification of genomic regions responsible for a related disease. We showed, however, that a successful identification of these regions can be accomplished without any prior knowledge of the species classification (Figure 5). Considering, in parallel, several hit region identification functions can provide more insight into the structure of genomic regions (Figures 4, 5 and 6). Simulation results suggest that there is no a unique function that provides the best overall results in all practical situations (e.g., the case of monophyletic or polyphyletic evolution and positive or negative selection), and that at least three different functions might be useful (Figures 2 and 3). It is worth noting that the monophyletic scenarios are easier to detect than the polyphyletic ones. The function $Q_{5}$ allows for a better detection of monophyletic scenarios, while in the polyphyletic case, the functions $Q_{4}$ and $Q_{6}$ provide the best results in the positive selection context and in the lineage specific selection context, respectively. The application of the described functions to the HPV gene E6 allows one to retrace the hit regions that are well-known to be related to carcinogenicity [26-28,30].

Furthermore, the results given by these functions while analyzing the FetA sequences of Neisseria meningitidis suggest a large overlap between the regions with surfaceexposed loops and those detected by the hit region identification functions (Figure 4). All these results indicate the ability of the proposed algorithm to identify regions with bipartite evolutionary signatures according to different patterns of evolution. Each time the species bipartition was known, High-Risk HPV against all other HPV types in Figure 5a, Squam-Risk HPV against all other HPV types in Figure 6a, and Adeno-Risk HPV against all other HPV types in Figure 6b, it was incorporated in the computational procedure as shown in Algorithm 1. In the future, it will be important to assess the correlation between different non-overlapping detected hit regions present in the given alignment. It would be also interesting to compare the performance of the introduced bi-clustering algorithm with the existing bi-clustering methods currently used in bioinformatics, including SAMBA [41], Crossing Minimization [42] and cMonkey [43]. Another possibility consists of using a $k$-means [44] type of algorithms that can suggest partitioning of the given dataset in several, and not necessarily in two, classes when the exact number of classes is unknown. For instance, in the case of HPV data, one could consider the three following HPV classes: High-Risk HPV (types 16 and 18), Low-Risk HPV (types 6, 11, 26, 31, $33,35,39,45,51,52,53,55,56,58,59,66,73,81,82$ and 83) and No-Risk HPV (all other HPV types).

It is worth noting that the presented algorithm, like most of the comparative genomics methods, relies on the assumption of the alignment correctness. Thus, it will be also important to analyze the impact of alignment errors on the results of the proposed hit detection procedure.

\section{Additional material}

\begin{abstract}
Additional file 1: Algorithm 1. Algorithm for computing genomic regions responsible for carcinogenicity or invasivity.p-values obtained for hit region detection using the remaining (i.e., not presented in Figs 2 and 3) $Q^{\prime}$-type functions $(a),(b),(c),(d)$

Monophyletic evolution - (e),(f),(g),(h) Polyphyletic evolution (a),(c),(e),(g) Positive selection - Variable hit region inside conserved context. Quartile distribution of p-values obtained for the functions

$Q_{5}^{\prime}(e), Q_{6}^{\prime}(c), Q_{5}^{\prime}(e)$, and $Q_{6}^{\prime}(g)$. Abscissa represents scaling factor of the conserved context in which the variable hit region resides. Values close to 0 represent conservation (maximum discrimination), while values close to 1 represent variability (identical to context). Variable hit region is always maintained at a scaling factor of 1 . Ordinate represents $\mathrm{p}$-values in log-scale. Horizontal dashed line represents the significance threshold of 0.05. (b),(d),(f),(h) Lineage specific selection - Heterogeneous hit region inside neutral context. Quartile distribution of $p$-values obtained for the functions $Q_{4}^{\prime}(b), Q_{6}^{\prime}(d), Q_{5}^{\prime}(f)$, and $Q_{4}^{\prime}(h)$ Abscissa represents the difference in scaling factors among the two lineages present in the hit region. Values close to 0 represent homogeneous evolutionnary speed (similar to the neutral context in which it resides), while values close to 1 represent divergence among these lineages. Context is always maintained at a scaling factor of 0.5 , simulating neutral evolution. Horizontal dashed line represents the significance threshold of 0.05 . In the case of lineage specific selection, the value of the $Q^{\prime}$-type functions corresponding to 1 on the abscissa scale cannot be computed because it involves a sub-tree with 0 edge lengths.
\end{abstract}

\section{Acknowledgements}

This study was funded by the Natural Sciences and Engineering Research Council of Canada (NSERC).

This article has been published as part of BMC Bioinformatics Volume 12 Supplement 9, 2011: Proceedings of the Ninth Annual Research in Computational Molecular Biology (RECOMB) Satellite Workshop on Comparative Genomics. The full contents of the supplement are available online at http://www.biomedcentral.com/1471-2105/12?issue=S9.

\section{Competing interests}

The authors declare that they have no competing interests.

Published: 5 October 2011

\section{References}

1. Posada D, Crandall K: Evaluation of methods for detecting recombination from DNA sequences: computer simulations. Proceedings of the National Academy of Sciences of the United States of America 2001, 98(24):13757.

2. Kimura M: The neutral theory of molecular evolution. Cambridge Univ Pr; 1985.

3. Boc A, Philippe $H$, Makarenkov V: Inferring and validating horizontal gene transfer events using bipartition dissimilarity. Systematic biology 2010, 59(2):195.

4. Moran P: The statistical processes of evolutionary theory. The statistical processes of evolutionary theory 1962.

5. Siepel A, Bejerano G, Pedersen JS, Hinrichs AS, Hou M, Rosenbloom K, Clawson H, Spieth J, Hillier LW, Richards S, Weinstock GM, Wilson RK, Gibbs Ra, Kent WJ, Miller W, Haussler D: Evolutionary conserved elements in vertebrate, insect, worm, and yeast genomes. Genome research 2005, 15(8):1034-50[http://www.ncbi.nlm.nih.gov/pubmed/16024819].

6. Yang Z: PAML: a program package for phylogenetic analysis by maximum likelihood. Comput. Appl. Biosci 1997, 13:555-556.

7. Yang Z, Nielsen R, Goldman N, Pedersen aM: Codon-substitution models for heterogeneous selection pressure at amino acid sites. Genetics 2000 
155:431-49[http://http: //www.pubmedcentral.nih.gov/articlerender.fcgi? artid $=1461088 \backslash \&$ tool $=$ pmcentrezl\&rendertype $=$ abstract] .

8. Yang Z: PAML 4: phylogenetic analysis by maximum likelihood. Molecular biology and evolution 2007, 24(8):1586.

9. Martin DP, Lemey P, Lott M, Moulton V, Posada D, Lefeuvre P: RDP3: a flexible and fast computer program for analyzing recombination. Bioinformatics (Oxford, England) 2010, 26(19):2462-3[http://http: //www pubmedcentral.nih.gov/articlerender.fcgi?artid=2944210l\&tool=pmcentrez I\&rendertype=abstract]

10. Milne I, Wright F, Rowe G, Marshall DF, Husmeier D, McGuire G: TOPALi: software for automatic identification of recombinant sequences within DNA multiple alignments. Bioinformatics (Oxford, England) 2004, 20(11):1806-7[http://www.ncbi.nlm.nih.gov/pubmed/14988107].

11. Badescu D, Diallo AB, Blanchette M, Makarenkov V: An Evolutionary Study of the Human Papillomavirus Genomes. Proceedings of RECOMB Comparative Genomics 2008, Volume 5267 of LNCS Springer; 2008.

12. Diallo AB, Badescu D, Blanchette M, Makarenkov V: A whole genome study and identification of specific carcinogenic regions of the human papilloma viruses. Journal of Computational Biology 2009, 16(10):1461-1473.

13. Badescu D, Diallo AB, Makarenkov V: Identification of Specific Genomic Regions Responsible for the Invasivity of Neisseria Meningitidis. In Classification as a Tool for Research. Springer Berlin Heidelberg;LocarekJunge H, Weihs C 2010:491-499.

14. Hubert L, Arable P: Comparing partitions. Journal of classification 1985, 2:193-218

15. Rand W: Objective criteria for the evaluation of clustering methods. Journal of the American Statistical association 1971, 66(336):846-850.

16. Jolley K, Chan MS, Maiden M: mlstdbNet - distributed multi-locus sequence typing (MLST) databases. BMC Bioinformatics 2004, 5:86[http:// www.biomedcentral.com/1471-2105/5/86].

17. Maiden M: Population genomics: diversity and virulence in the Neisseria. Current opinion in microbiology 2008, 11(5):467-471.

18. Pettersson A, Poolman J, van der Ley P, Tommassen J: Response of Neisseria meningitidis to iron limitation. Antonie van Leeuwenhoek 1997, 71:129-136.

19. Pettersson A, Maas A, Van Wassenaar D, Van der Ley $P$, Tommassen J: Molecular characterization of $\mathrm{FrpB}$, the 70-kilodalton iron-regulated outer membrane protein of Neisseria meningitidis. Infection and immunity 1995, 63(10):4181.

20. Kortekaas J, Pettersson A, Van der Biezen J, Weynants V, Van der Ley P, Poolman J, Bos M, Tommassen J: Shielding of immunogenic domains in Neisseria meningitidis FrpB (FetA) by the major variable region. Vaccine 2007, 25:72-84.

21. Urwin R, Russell J, Thompson E, Holmes E, Feavers I, Maiden M: Distribution of surface protein variants among hyperinvasive meningococci: implications for vaccine design. Infection and immunity 2004, 72(10):5955.

22. Altschul S, Gish W, Miller W, Myers E, Lipman D: Basic local alignment search tool. Journal of molecular biology 1990, 215(3):403-410.

23. Angulo M, Carvajal-Rodríguez A: Evidence of recombination within human alpha-papillomavirus. Virol J 2007, 4:33.

24. Bosch F, Manos M, Muñoz N, Sherman M, Jansen A, Peto J, Schiffman M, Moreno V, Kurman R, Shan K: Prevalence of human papillomavirus in cervical cancer: a worldwide perspective. Journal of the National Cancer Institute 1995, 87(11):796.

25. Munoz N: Human papillomavirus and cancer: the epidemiological evidence. Journal of clinical virology 2000, 19(1-2):1-5.

26. Lee C, Laimins L: Role of the PDZ domain-binding motif of the oncoprotein E6 in the pathogenesis of human papillomavirus type 31. Journal of virology 2004, 78(22):12366.

27. Kiyono T, Hiraiwa A, Fujita M, Hayashi Y, Akiyama T, Ishibashi M: Binding of high-risk human papillomavirus E6 oncoproteins to the human homologue of the Drosophila discs large tumor suppressor protein. Proceedings of the National Academy of Sciences of the United States of America 1997, 94(21):11612.

28. Lipari F, McGibbon G, Wardrop E, Cordingley M: Purification and biophysical characterization of a minimal functional domain and of an $\mathrm{N}$-terminal $\mathrm{Zn2+-binding} \mathrm{fragment} \mathrm{from} \mathrm{the} \mathrm{human} \mathrm{papillomavirus} \mathrm{type}$ 16 E6 protein. Biochemistry 2001, 40(5):1196-1204.

29. Nominé $Y$, Charbonnier S, Ristriani T, Stier G, Masson M, Cavusoglu N, Van Dorsselaer A, Weiss E, Kieffer B, Travé G: Domain substructure of HPV E6 oncoprotein: biophysical characterization of the E6 C-terminal DNAbinding domain. Biochemistry 2003, 42(17):4909-4917.

30. Nominé Y, Masson M, Charbonnier S, Zanier K, Ristriani T, Deryckère F, Sibler A, Desplancq D, Atkinson R, Weiss E, et al: Structural and functional analysis of E6 oncoprotein: insights in the molecular pathways of human papillomavirus-mediated pathogenesis. Molecular cell 2006, 21(5):665-678.

31. Munoz N, Bosch F, de Sanjose S, Herrero R, Castellsagué X, Shah K, Snijders P, Meijer C: Epidemiologic classification of human papillomavirus types associated with cervical cancer. New England Journal of Medicine 2003, 348(6):518-527.

32. Munoz N, Bosch F, Castellsagué X, Díaz M, de Sanjose S, Hammouda D, Shah K, Meijer C: Against which human papillomavirus types shall we vaccinate and screen? The international perspective. International Journal of Cancer 2004, 111(2):278-285.

33. Chan S, Delius H, Halpern A, Bernard H: Analysis of genomic sequences of 95 papillomavirus types: uniting typing, phylogeny, and taxonomy. Journal of virology 1995, 69(5):3074.

34. Milligan G, Cooper M: A study of the comparability of external criteria for hierarchical cluster analysis. Multivariate Behavioral Research 1986.

35. Santos J, Embrechts M: On the use of the adjusted rand index as a metric for evaluating supervised classification. Artificial Neural NetworksICANN 2009, 175-184.

36. Kuhner M, Felsenstein J: A simulation comparison of phylogeny algorithms under equal and unequal evolutionary rates. Molecular Biology and Evolution 1994, 11(3):459.

37. Guindon S, Gascuel O: Efficient biased estimation of evolutionary distances when substitution rates vary across sites. Molecular biology and evolution 2002, 19(4):534

38. Rambaut A, Grass N: Seq-Gen: an application for the Monte Carlo simulation of DNA sequence evolution along phylogenetic trees. Computer applications in the biosciences: CABIOS 1997, 13(3):235.

39. Jukes T, Cantor C: Evolution of protein molecules. Mammalian Protein Metabolism Academic Press, New York; 1969, 21-132.

40. Van Der Ley P, Heckels J, Virji M, Hoogerhout P, Poolman J: Topology of outer membrane porins in pathogenic Neisseria spp. Infection and immunity 1991, 59(9):2963.

41. Tanay A, Sharan R, Kupiec M, Shamir R: Revealing modularity and organization in the yeast molecular network by integrated analysis of highly heterogeneous genomewide data. Proceedings of the National Academy of Sciences 2004, 9:2981-2986.

42. Abdullah $A$, Hussain $A$ : A new biclustering technique based on crossing minimization. Neurocomputing 2006, 69:1882-1896.

43. Reiss D, Baliga N, Bonneau R: Integrated biclustering of heterogeneous genome-wide datasets for the inference of global regulatory networks. BMC Bioinformatics 2006, 7:280.

44. MacQueen J: Some Methods for classification and Analysis of Multivariate Observations. Proceedings of 5th Berkeley Symposium on Mathematical Statistics and Probability 1967, 2:281-297.

doi:10.1186/1471-2105-12-S9-S9

Cite this article as: Badescu et al.: Detecting genomic regions associated with a disease using variability functions and Adjusted Rand Index. BMC Bioinformatics 2011 12(Suppl 9):S9.

\section{Submit your next manuscript to BioMed Central and take full advantage of:}

- Convenient online submission

- Thorough peer review

- No space constraints or color figure charges

- Immediate publication on acceptance

- Inclusion in PubMed, CAS, Scopus and Google Scholar

- Research which is freely available for redistribution 